\title{
Nutritional well-being of young children in Duncan Village, East London, South Africa: accessibility of primary health care clinics
}

\author{
Anniza de Villiers ${ }^{1}$, Kholeka Koko-Mhlahlo ${ }^{2}$ and Marjanne Senekal ${ }^{1, *}$ \\ 'Department of Physiological Sciences, Stellenbosch University, Private Bag X1, Matieland 7602, South Africa: \\ ${ }^{2}$ Department of Social Work, Stellenbosch University, South Africa
}

Submitted 15 May 2005: Accepted 15 June 2005

\begin{abstract}
Objective: The aim was to contribute to the nutritional well-being of young children living in Duncan Village by investigating factors that influence clinic attendance of mothers and to formulate recommendations for optimisation of accessibility of primary health care (PHC) clinics in the area.

Design: PHC clinic accessibility was evaluated by assessing the experiences of mothers who attended clinics in the area as well as the experiences of health care workers (HCWs) in these clinics of service delivery and its recipients (mothers/children), using the focus group technique. The ATLAS/ti program was used to analyse the data in the following steps: preparation and importing of the data, getting to know and coding the data, retrieval and examination of codes and quotations, creation of families and creation of networks.

Setting: Duncan Village, a low socio-economic urban settlement in East London, South Africa.

Subjects: Focus group discussions (four to seven participants per group) were conducted with four groups of mothers who do not attend PHC clinics, six with mothers who do attend the clinics (including pregnant women) and four groups of HCWs.

Results: Four networks that provide a summary of all the major trends in the data were created. The results clearly indicate that mothers in Duncan Village perceive and/or experience serious problems that make it difficult for them to attend clinic and even prevent them from doing so. These problems include both the way they are treated at the clinics (especially the problem of verbal abuse) as well as the actual services delivered (no medicines, no help, disorganised, long waiting periods, being turned away). The main problem experienced by the HCWs with service delivery seems to be a heavy workload, as well as the fact that many mothers do not come for follow-up visits.

Conclusion: Efforts to increase the accessibility of PHC clinics in Duncan Village should focus on improving the relationship between mothers and HCWs and the heavy workload experienced by these workers.
\end{abstract}

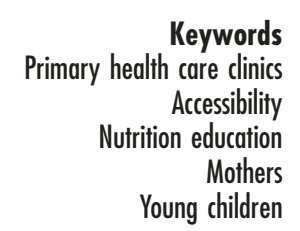

In 2002, the World Health Organization (WHO) noted that the world had witnessed a remarkable achievement: 'Child mortality has decreased from 97 per 1000 live births in the early 1980s to 67 per 1000 live births in 1999'1. Effective public health interventions delivered to large numbers of children were identified by the WHO as being responsible for the major part of this success ${ }^{1}$. Despite this apparent success, the prevailing situation is still unacceptable.

According to the Fourth Report on the World Nutrition Situation of the United Nations Standing Committee on Nutrition, $32.5 \%$ of pre-school children in developing countries were stunted at the beginning of the new century and $26.7 \%$ were underweight. Sub-Saharan Africa has seen the least overall improvement in the nutritional status of its pre-school children ${ }^{2}$.

In South Africa, a national survey undertaken in 1994 by the South African Vitamin A Consultative Group (SAVACG) found that stunting was a major problem, with one in four children being stunted ${ }^{3}$. Two of the key recommendations of the SAVACG were the targeting of the very young $(<2$ years) for nutrition intervention as well as the targeting of mothers for nutrition education, socio-economic upliftment, income generation and child care. Five years later (1999) the National Food Consumption Survey (NFCS) was 
undertaken and found nationally that one out of five children was stunted and one out of 10 was underweight ${ }^{4}$. Undernutrition therefore remains a serious health problem among many South African children.

The Eastern Cape Province of South Africa, where the present research was conducted, is one of the worst-off areas in the country as far as health and socio-economic indicators are concerned ${ }^{5}$. The 1999 NFCS did indeed identify the Eastern Cape province as one of the four most seriously affected provinces in relation to nutrition ${ }^{4}$.

Duncan Village, the densely populated urban residential area on which the research focused, is situated in one of the poorest municipalities in the area ${ }^{6}$. Bank et $a l^{7}$ describe Duncan Village as a 'deeply impoverished urban slum'. The 1996 census data (personal communication; Swart, Buffalo City Municipality Planning Department, 2003) showed that only $23 \%$ of the population of Duncan Village is employed, $18 \%$ of people have no education at all, and most people still live without electricity and running water in their houses or proper ablution facilities. Although poor environmental conditions do not imply that health impairment will take place, children are especially susceptible to disease if they are born in such environments. They not only suffer from the direct consequences of the environment, but also from the stress and other psychosocial factors that such environments create for them and their caregivers ${ }^{8}$.

Various initiatives are taking place to address nutrition problems in South Africa through the government's Integrated Nutrition Programme, programmes of the Health Systems Trust and other initiatives such as the Transnet-Phelophepa Health Care Train. However, very few of these initiatives are available to households in Duncan Village (personal communication; Mdingi, Head, Regional Office, Sub-directorate Nutrition, Province of the Eastern Cape, 2000). The need for clearly targeted intervention initiatives to ensure the nutritional health of young children in Duncan Village was clearly evident.

To address this need the Triple A process, a participatory decision-making process pioneered by the United Nations Children's Fund (UNICEF) ${ }^{9}$, was initiated. As a first step in the process, a case-control study was undertaken from a health centre in Duncan Village to identify determinants of growth failure in 12- to 24-month-old children attending the centre. Specific priorities for initiatives to address the problem in the area were subsequently identified ${ }^{10}$. These priorities were clearly in line with the priorities of early childhood as set out in the UNICEF medium-term strategic plan for 2002-2005; i.e. girls' education, integrated early childhood development, immunisation, fighting HIV/ AIDS and improved protection of children from violence, abuse, exploitation and discrimination ${ }^{11}$. They were also in line with the six elements of UNICEF's 'Care Initiative', i.e. care for women, breast-feeding and feeding practices, psychosocial care, food preparation, hygiene practices and home health practices ${ }^{12}$.
An important point for consideration in intervention planning involves ensuring the availability of the necessary infrastructure for implementation ${ }^{13}$. After wide investigation and consultation, it was decided that the existing infrastructure of the municipality clinics could provide a sustainable vehicle for providing nutritionrelated education. As the first-level primary health care (PHC) structures in Duncan Village, the clinics would be the most likely place where mothers or pregnant women would be exposed to any interventions, i.e. at antenatal clinics or at the immunisation clinics. This is supported by the comment of Shearley ${ }^{14}$ that vaccination programmes are the only recurring activity in primary health care that bring mothers and children into contact with health services on a predictable and frequent basis, providing an opportunity for family health education.

Unfortunately there is some concern about the accessibility of PHC services to certain sectors of the Duncan Village population. It has been noted by $\mathrm{UNICEF}^{15}$ that high-risk groups - generally the poorest - are often marginalised from society as a whole, including the health care system, and usually cannot be reached unless a deliberate effort is made. Hence it was argued that a consultative approach to assess the accessibility of PHC clinics in the area, as a further step in the assessment and analysis phases of the Triple A process, would contribute to sustainable action to ensure the nutritional well-being of young children in Duncan Village. Therefore the aim of the present research was to contribute to the nutritional well-being of young children living in Duncan Village by investigating factors that influence clinic attendance by mothers and to formulate recommendations for optimisation of accessibility of PHC clinics in the area.

\section{Methods}

To determine the accessibility of PHC clinics in Duncan Village, the experiences of mothers living in the catchment area of the PHC clinics, as well as the experiences of the personnel of these clinics of service delivery and its recipients (mothers/children), were assessed. The nature of these objectives was such that group interviews, where group members could freely discuss their experiences and feelings, as is the case in focus group discussions, were considered ideal. The 'design by dialogue' process suggested by Dicken et al. ${ }^{16}$ was used as the starting point to structure the research.

\section{Study population, sample size and sampling}

As recommended by Dicken et al. ${ }^{16}$, the selection of a sample for consultative research entailed the identification of population units, research sites and participant categories. Women attending the clinics in Duncan Village were identified as population units. Buffalo City Municipality and Frere Hospital were selected as research sites, 
because both these two institutions include staff who worked directly with mothers from the population units. Subsequently four participant categories were selected for the research. The different categories, the selection criteria and the number of planned and actual focus groups conducted are summarised in Table 1.

As it was important to provide an environment where participants would be able to express their feelings and opinions freely, a convenient site away from the clinics was selected to conduct the focus groups with the mothers.

\section{Focus group size}

Although Fern ${ }^{17}$ recommends the inclusion of 10 to 12 people per focus group, the number of participants in the groups in this study varied between four and seven. Large groups of 10 to 12 were impractical because of small children attending the groups with their mothers. In the case of the health care workers (HCWs), the small study population restricted the availability of a large group of participants.

\section{Number of focus groups}

Fern $^{17}$ recommends four to six groups per 'break characteristic'. The latter was defined as 'whether mothers attend clinics in Duncan Village or not' for the purposes of this study. Four focus groups were conducted with mothers who do not attend clinic, six with mothers who attend clinic (including pregnant women) and four with health workers (Table 1).

\section{Sampling}

Participants for the mother and pregnant women focus groups were selected directly from the community and not through any of the health structures. Separating the sampling process as well as the venue from the clinics ensured that participants could express their views more freely without any fear of possible negative consequences. The 'reliance on available subjects' technique combined with snowball sampling was used to select participants for the mother and child categories ${ }^{18}$. For identification of participants in the health worker categories, the clinic managers selected staff members available at the particular time. The potential number of participants in these categories was relatively small and all available were included.

\section{Research instruments}

\section{Research protocol}

A research protocol was compiled that provided guidance to the co-ordinator on each step of the research process. The research protocol also served as the procedure manual and was used to train the co-ordinator and the fieldworker.

Table 1 Categories of participants

\section{Category A: Biological mothers who do not attend clinic/mothers with children with incomplete immunisations}

Mothers with a child

$<10$ months old

Mothers with a child $>10$ and $<20$ months old

\section{Mothers with one child only} who is $>10$ and $<20$ months old

Mothers with a child $>10$ and $<20$ months old

Mother has more than one child; youngest $<10$ months old. Child did not attend clinic at all or missed any one of the three compulsory immunisations in the first 10 months

Mother has more than one child; any one of the children could be between 10 months and 20 months old. The specific child did not attend clinic at all or missed any one or more compulsory immunisations in the first 20 months Mother has only one child. This child is younger than 20 months and did not attend clinic at all or missed any one or more compulsory immunisations in the first 20 months

Mother left Duncan Village clinics to attend clinic elsewhere

\section{Category B: Biological mothers with children who have been immunised completely}

Mothers with a child $<10$ months Mother has more than one child; youngest $<10$ months old. The child must old

Mothers with child

Mothers with only one child who is $>10$ and $<20$ months old

\section{Category C: Pregnant women}

Pregnant women who attend antenatal clinic

Pregnant women who do not attend antenatal clinic

Category D: Health workers

Duncan Village municipality clinics Frere Hospital maternity wards Community health workers have a clinic card and must be fully immunised

Mother has more than one child; any one of the children must be between 10 and 20 months old. The child must have a clinic card and must be fully immunised Mothers must have only one child. The child must be between 10 and 20

The participant must have kept to most $(90 \%)$ of her antenatal clinic dates

The participant must not have attended antenatal clinic at all or have attended but stopped attending

Nursing professionals working at the clinics

Any level of nursing staff dealing with mothers in the obstetric unit Community health workers employed by the municipality 


\section{Focus group guides}

The aim of the study, namely the determination of the accessibility of PHC clinics in Duncan Village, guided the development of the focus group guides. Questions about experiences with the clinic, difficulties in attending clinic and benefits gained from attending clinic were included for mothers and pregnant women. Questions concerning feelings towards clients and the work constraints encountered were included for the HCWs. All categories were asked for recommendations on what could be done to 'make it easy for mothers to attend clinic'.

\section{Fieldworkers}

A Xhosa-speaking social worker with three children of her own, a good knowledge of the target community and appropriate experience in qualitative research methodology was appointed to moderate the focus group discussions. A mature woman from Duncan Village assisted with sampling and the practical arrangements of the focus groups. Both these fieldworkers were specifically trained on the sampling techniques and the management of the focus groups for this study.

\section{Data collection}

Data were collected during a one-week period. All focus group discussions were conducted in Xhosa, the indigenous language of the area, except the two conducted with health workers at Frere Hospital, which were conducted in English. Discussions were taperecorded and debriefing sessions with the moderator and the fieldworker were conducted at the end of each focus group session.

\section{Data analysis}

A systematic approach was used to analyse the data, using the ATLAS $/ \mathrm{ti}^{19}$ program. The following steps were executed in this process.

\section{Preparing and importing the data}

Tape-recorded focus group discussions were transcribed and translated into English (in one step) by a professional transcriber. For auditing purposes one of the focus groups was first transcribed into Xhosa and then translated from Xhosa into English. The 14 transcriptions were loaded into ATLAS/ti as 14 primary documents.

\section{Getting to know the data}

This involved thorough reading of the transcriptions of the focus group discussions to obtain insight into the data.

\section{Coding the data}

Descriptive coding of the data was carried out, which involved classification of a selected segment of textual data by means of a label (summary term) that expresses some essential quality of the phenomenon as reflected in the data. An inductive approach according to which codes are derived from the data and not from a pre-determined list of codes was followed in this process, which facilitates the discovery of categories and patterns within the data. A preliminary code list was developed that was used to revisit the data, resulting in further refinement of the code list. A continuous revisiting of the data is necessary as additional codes emerge during the coding process. This process of refinement was continued until a final code list was achieved.

\section{Retrieval and examination of codes and quotations}

This involved revisiting the data again with the final code list and codes, and the retrieval of related quotations in order to examine patterns and trends in the data.

\section{Creation of families}

Families are 'containers' for primary documents and codes that are of the same type ${ }^{19}$. The main objective of the creation of families is to cope with a possibly large number of objects by classifying them into sub-sets. For our data analysis two primary document families were created, one for mothers and one for HCWs. Subsequently the codes were grouped into the 12 code families presented in Table 2 .

\section{Creation of networks}

This involved the creation of networks that are the main ingredients for constructing theoretical models with ATLAS $/ \mathrm{ti}^{19}$ and allowed the creation of visual images of the links in the data to illustrate interrelationships ${ }^{18}$.

\section{Validity}

Measures that were taken to ensure design validity and objectivity of the data are presented in Table 3.

\section{Ethical issues}

Ethical permission for the research was obtained from the ethics committee at Frere Hospital and permission to access clinic staff was obtained from the Buffalo City Municipality. Oral consent for participation was obtained from each potential participant.

\section{Results}

Each of the 12 code families listed in Table 2 were written up in detail. For the purposes of this paper four networks (Figs 1-4) that provide a summary of all the major trends in the data were created and are discussed. Guidelines for the interpretation of the networks are provided in Table 4.

Network 1 (Fig. 1) presents the interpersonal aspects (between client and health worker) identified by mothers that either make it difficult for them to attend clinic or cause them not to attend clinic at all. Verbal abuse by nurses is a direct cause $(\Rightarrow)$ of non-attendance. Verbal abuse is also a direct cause of fear (afraid), which in turn is 
Table 2 Primary document (PD) families and codes

\begin{tabular}{|c|c|c|c|c|}
\hline PD family & No. of PDs & Code families & $\begin{array}{c}\text { Abbreviation } \\
\text { in networks } \\
\text { (Figs 1-4) }\end{array}$ & $\begin{array}{l}\text { No. of } \\
\text { codes }\end{array}$ \\
\hline \multirow[t]{8}{*}{ Health care workers (HCWs) } & 4 & Difficulties experienced by HCWs & HCWdif & 8 \\
\hline & & HCWs - Experiences with the community & HCWExCom & 9 \\
\hline & & HCWs - Experiences with the mothers & HCWExMoth & 13 \\
\hline & & HCWs - Coverage of the messages & HCWcov & 9 \\
\hline & & HCWs - Recommendations & HCWrec & 13 \\
\hline & & Clinic & HCWrecClin & \\
\hline & & Education & HCWrecEd & \\
\hline & & Mothers & HCWrecMoth & \\
\hline \multirow[t]{9}{*}{ Mothers and pregnant women } & 10 & Difficulties experienced by mothers & Mdif & 7 \\
\hline & & Mothers' perceptions & MP & 2 \\
\hline & & Benefits & M & 3 \\
\hline & & Mothers' feelings & MF & 9 \\
\hline & & Mothers' experiences with the clinic staff & MExNurses & 9 \\
\hline & & Mothers' experiences with services received & MExSR & 10 \\
\hline & & Mothers' recommendations & & 12 \\
\hline & & Health services & MrecHS & \\
\hline & & Nurses & MrecNurses & \\
\hline Total & 14 & & & 104 \\
\hline
\end{tabular}

Table 3 Objectivity and design validity criteria for qualitative research

\begin{tabular}{ll}
\hline Design validity criteria $^{18,20}$ & Application in this research
\end{tabular}

Credibility: Compatibility between 'constructed realities in the minds of the respondents' and those that are attributed to them Prolonged engagement: was the observation long enough to provide an accurate picture? The primary researcher worked in the health system in the community for more than 10 years and the current research evolved from the case-control study ${ }^{10}$; therefore involvement with this research extended over a prolonged period (15 years)

Persistent observation: was sufficient time spent on site to get an adequate picture of consistency of behaviour?

The fieldwork of the current research was executed over a 10-day period. As this was based on the culmination of 8 years of work concerning the nutritional well-being of young children in Duncan Village, the 10 days allowed sufficient time 'on site' to obtain an adequate picture of the consistency of 'experiences'

Debriefing interviews were conducted with fieldworkers after focus group discussions, which allowed the expression of different viewpoints on whether experiences of participants had been discussed thoroughly enough to allow conclusions to be made about the consistency thereof

Triangulation: were datasets/perspectives combined An expert in nutrition studied the interpretations thoroughly and provided an additional perspective where necessary

Relevant literature was identified and integrated with the data for formulation Referential adequacy: was enough supportive material used?

Peer debriefing: was another professional consulted for additional perspectives? of conclusions on 'accessibility' of PHC clinics An expert in qualitative data methodology and analysis provided critique on methodological and data analysis issues. An expert in nutrition provided critique on the data interpretation within the nutrition context

Transferability: Extent to which findings can be applied to other contexts or respondents

Thick description: was sufficient detail recorded and reported?

Purposive sampling: were locations and informants selected that differ from each other enough to maximise the range of information obtained? Structural relationships: were different datasets interwoven during data interpretation?

Dependability: A measure of the reliability of data Inquiry audit: were the documents examined by an auditor?

\section{Confirmability: Refers to potential use/replication of data/results by others}

Audit trail: is good documentation available for use by or replication by other researchers?
Data were analysed and reported with as much detail and precision as was possible using the CAQDAS, ATLAS/ti. The context of the Duncan Village situation was borne in mind at all times

Participant categories were carefully selected to ensure wide coverage. The sampling process ensured the greatest representativeness possible in the different categories

Findings of the different participant categories were interpreted and interwoven to present a proposed framework for accessibility of nutrition-related education at the PHC clinics in Duncan Village

Two of the researchers completed a thorough inquiry audit 


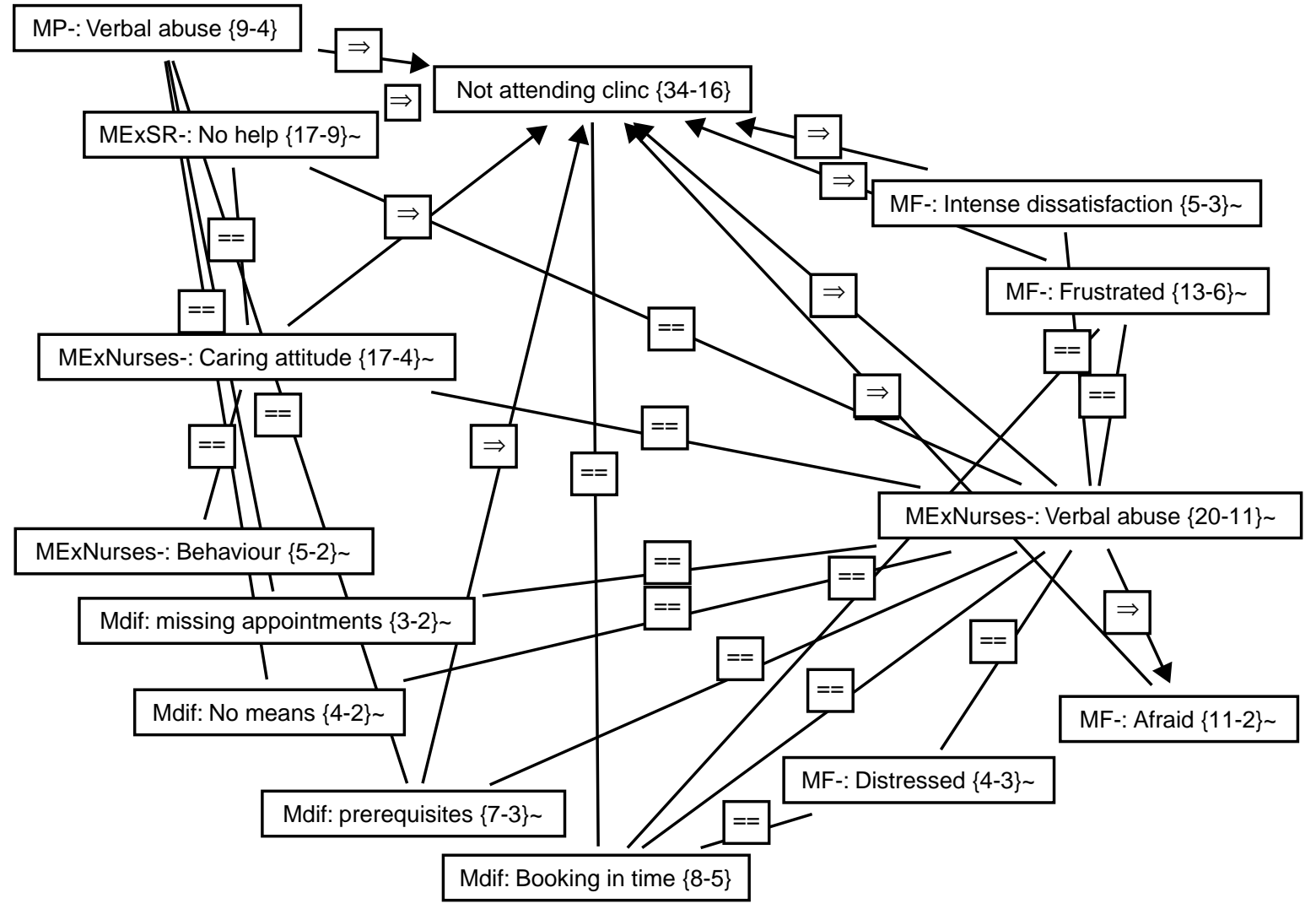

Fig. 1 Interpersonal aspects (between mothers and health care workers) identified by mothers that influence clinic attendance

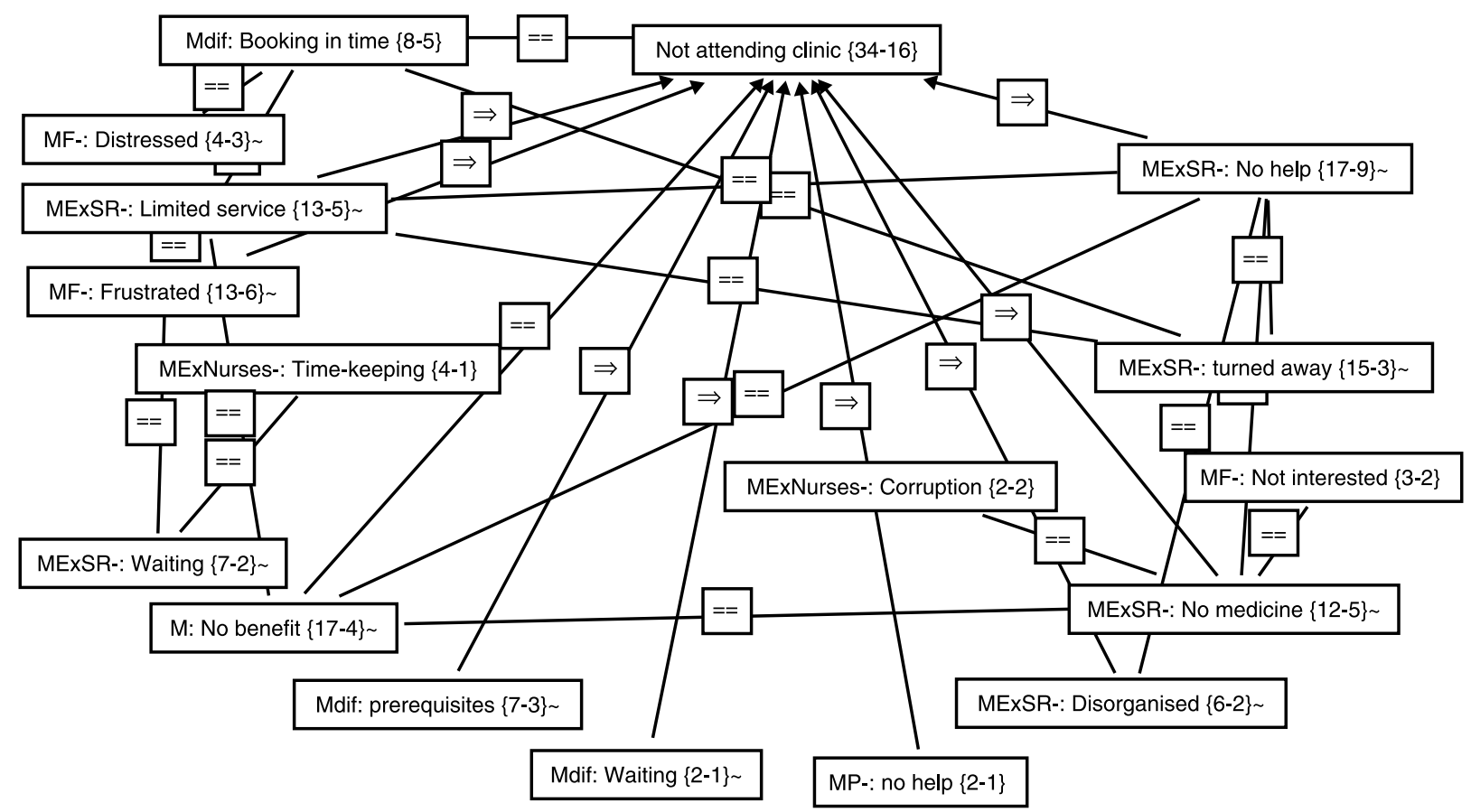

Fig. 2 Aspects regarding the day-to-day management of clinics identified by mothers as factors that influence clinic attendance

a direct cause of non-attendance. Verbal abuse is also associated $(==$ ) with frustration, intense dissatisfaction, being distressed, booking time, prerequisites (ID and clinic card), no means (no money to feed and keep baby clean and properly dressed), missing appointments, poor caring attitude of HCWs and no help received when the clinic was visited. These associations indicate that most of the mothers' perceptions, feelings and experiences 


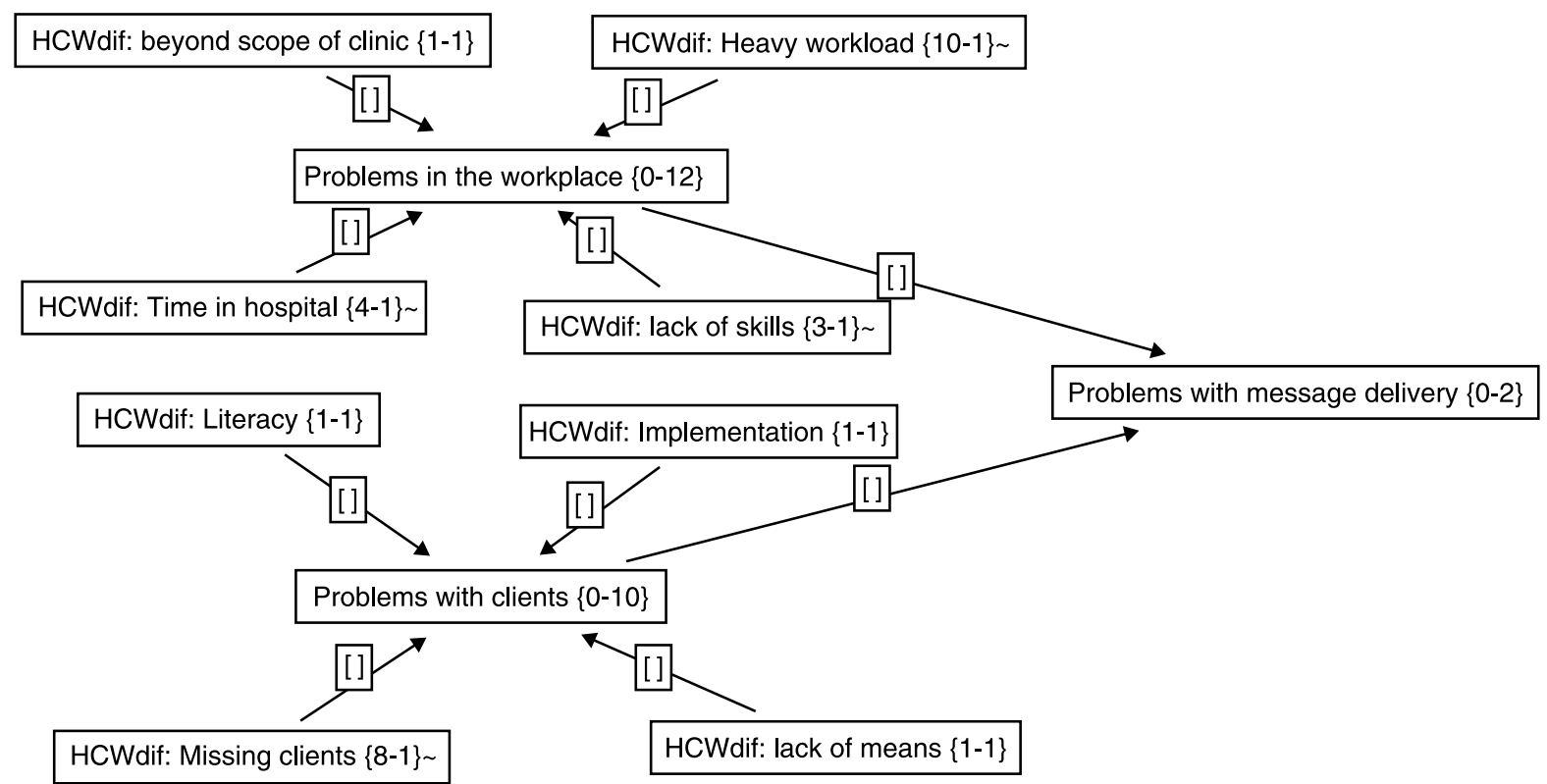

Fig. 3 Factors influencing the quality of service delivery by health care workers

associated with non-attendance have their roots in verbal abuse by HCWs. Other interpersonal aspects that seem to be direct causes of non-attendance include no help received, poor caring attitude of the HCWs, frustration, prerequisites and finally intense dissatisfaction. Each of these is in turn associated with one or more of the other codes included in the network. The following quotes from the relevant primary documents illustrate the abovementioned perceptions, feelings and experiences of mothers:

The nurses shout at us. This makes us not to go to the clinic. The child ends up not immunised.'

It is painful because I went to the clinic with my baby who burned. I was hysterical; I took the child to the clinic. The nurses shouted, swore and insulted me. They send me away saying that the clinic is not a place for burnt children. I have boped that the nurses would help me. From that day I lost interest and I decided that I would never go to the clinic again.'

'The nurses always shout at us saying we have to feed the babies because they are working on their nerves when they cry. That time you do not have anything to feed the baby. So I decide to stay bome.'

'When you come back they tell you that the number is enough for the day come another day. A person has to go back bome. Sometimes the child is sick. You go home without help.'

'They offend us about our dirty babies. They do not even know your background. Most of the time we do not even have money to buy soap to wash the baby clothes and nappies. They will offend us about the greyish clothes and nappies.'
I lost interest because if the child is sick to death the nurses will give you Panado, even if the child has to be referred to the hospital.'

'The nurses do not have a way of talking, they do not respect us and they do not care. I am tired of them.'

Network 2 (Fig. 2) presents the organisational aspects regarding the day-to-day management of clinics identified by mothers as factors that contribute to non-attendance. Frustration, the perception/experience of no benefit gained and not getting any help, prerequisites, disorganised services, long waiting periods, and no medicine available are all direct causes $(\Rightarrow)$ of mothers not attending the clinics. Many of these are also indirectly linked to non-attendance; for example, no benefit in attending is associated $(==$ ) with a lack of medicine and no help received. Not receiving any help is in turn associated with the service being disorganised, being turned away, limited service (e.g. no clinic services available on a Friday) and no medicine. No medicine available is associated with corruption, no help available and feelings of not being interested in attending the clinic. The following quotes from the relevant primary documents illustrate the above-mentioned perceptions, feelings and experiences of mothers:

I decided not to go to the clinic because a person does not get belp. A person is always turned away because there are no medicines, not even for immunisation.'

'I become frustrated because I do not understand why they say it is a clinic when there is always no medicines.'

Medicines are given to certain individuals but the nurses always say there are no medicines. Nurses take medicines to their home.' 


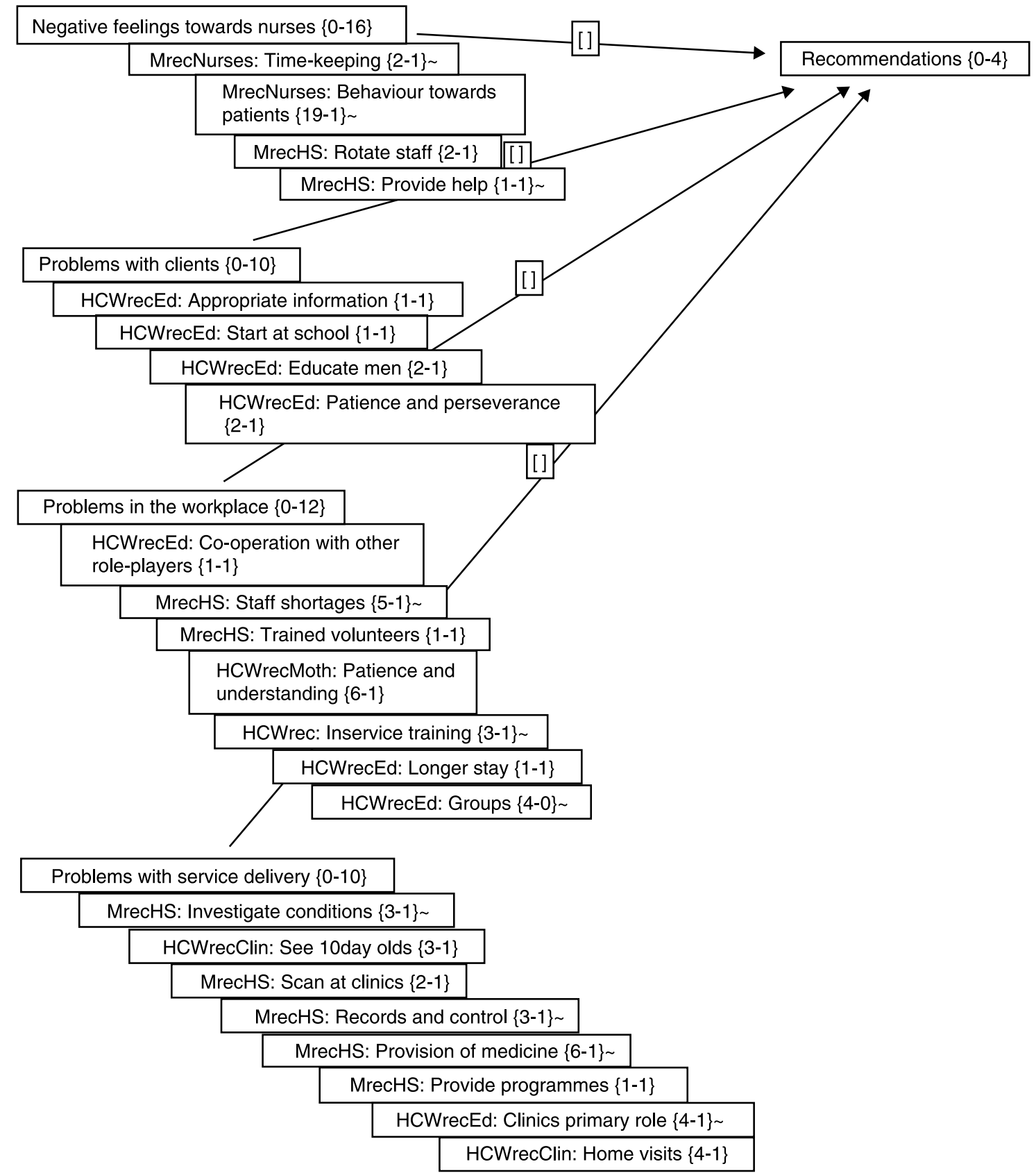

Fig. 4 Recommendations made by both health care workers and mothers to ensure and improve clinic attendance

Table 4 Guidelines for the interpretation of ATLAS/ti networks

Code label: The code label MP: Verbal abuse $\{9-4\}$ is used as an example:

The family abbreviation MP refers to the code family: Perceptions of mothers (see Table 2 for explanation of other abbreviations)

This is followed by the code developed to describe a salient quotation in this case 'verbal abuse'

The figures in brackets that follow the code indicate the number of times a particular code occurred ( 9

in this example), followed by existing linkages to other codes (4 in this example). If the first figure in the brackets is 0 , it indicates that the code has been created specifically for the network and does not have any quotations attached to it

Links: The lines between codes are links that indicate a relationship between the codes. The type of relationship could be defined by a symbol being placed on the link: e.g. $==$ indicates that Code 1 is associated with Code 2, [] indicates that Code1 is part of Code 2 and $\Rightarrow$ that Code 1 is the cause of Code 2 
'Fridays they send you back because they do admin. They will not help you even if your child is sick.'

'Nurses are always on the phone. They attend you after spending a lot of time on the phone.'

'The nurses tell you to be on time for immunisation. You come at 8 am the gates are not open yet. When you are inside they spend another half-hour preparing, others having tea. You leave your home early because you do not want to be insulted for being late.'

'They do favours. They will attend to someone they know even if you came earlier than she did. This makes you cheeky and not to come to the clinic again.'

It was not possible to create a meaningful network based on interpersonal organisational aspects identified by the mothers that either makes it easy or attractive for them to attend clinic, as the number of such responses was too low. However, to ensure that a complete picture is provided, quotations that reflect more positive experiences are included, as follows:

'From the clinic I get advice from other mothers about feeding the baby, how to care for the nappies, etc.'

'I feel bad because some nurses are good and some are bad.'

'There is belp but not sufficient.'

'To my surprise they educated us on how to care for the child.'

'It helps because they test you for HIV/AIDS so that if you are positive they give you Neviropine.'

If feel good sometimes. I had a problem with my pregnancy and they referred me to the hospital. If I did not go I would not have known what was wrong.'

Network 3 (Fig. 3) shows that factors influencing the quality of service delivery by HCWs (as mentioned by the HCWs themselves) involve problems in the workplace as well as problems with clients (mothers). Problems in the workplace seem to be mainly linked ([]) to a heavy workload and the short time period available in the obstetric unit for education. The fact that the health problem experienced by a particular mother was at times beyond the scope of competence of HCWs and that they did not have the skills to handle all problems were also mentioned. The most prominent link ([]) to problems HCWs experience with clients seems to be the fact that the clients do not return to the clinic (missing clients). Poor household food security (no means), illiteracy and mothers not implementing advice given to them were also linked to the code: problems with clients. The following quotes from the relevant primary documents illustrate above-mentioned factors that influence the quality of service delivery by HCWs:

'We are only two sisters in my clinic. We do tell the women how many we are going to take for the day. We will not be able to take more.'
'I would tell them what I know. I don't feel it is enough.' (lack of knowledge on the part of the HCWs)

'Sometimes non-availability of resources is a problem. Sometimes you want to refer the mother, because you feel the problem is beyond your scope of practice.'

'We do educate them on what to do, but they do not have the means to buy the things they need for the babies.'

'After two years, they come back for a clinic card saying they want to register their babies. Every time they are discharged, we give them a lecture on what to do about clinic cards and the form for registering the baby.'

'They migrate to urban areas when they are pregnant. After the delivery they go back. When you do follow up you find that she is no longer here - she gone to Transkei. When they move, they do not inform the clinics.'

'After giving birth they sometimes do not come back to us. Alternatively, they will come back with the malnourished 6month-old babies.'

'Few people do implement what they have been educated about. We do educate people on bealth but it takes time for them to do what they have been told.'

'The other problem is that they believe too much in witch doctors. You find that when they come back to the clinic the child is another state. When you ask why, they will tell you that she was using traditional healer's medicine.'

'You will educate her and she will tell you what her motherin-law said, so you find there is conflict of ideas.'

'The teenagers do not want to breast-feed. They do not want to come to the nursery. They will only come out to see the child when there is a relative or a boyfriend.'

Network 4 (Fig. 4) displays the recommendations made by both HCWs and mothers to ensure and improve clinic attendance. Most recommendations formulated by mothers concern addressing the negative feelings they have towards the HCWs. Mothers want nurses to treat them with respect by talking to them respectfully, respecting their thoughts, opinions and privacy; communicating with them properly; not projecting their own problems on the clients; being sensitive to their circumstances (hygiene aspects and poverty); and by being ethical and fair in their dealings with clients. As far as the organisation of the clinics is concerned, mothers would like the situation to be investigated, medicine availability to improve, staff shortages to be addressed and the booking system to be improved, so that they do not go and then get turned away after hours of waiting. The following quotes from the relevant primary documents illustrate above-mentioned recommendations by mothers:

If the nurses can show some respect and be able to talk to us as human beings and show that they care, I will go to the clinic again.' 
'The nurse must learn to trust us when we tell them we have a problem.'

'They must have confidentiality. We even know some patients who are HIV-positive, but that is suppose to be confidential.'

'Nurses need to be trained on how to talk to people.'

'Nurses must stop taking their frustrations out on us.'

'Nurses must stop doing favours. They must use the first come first served policy.'

'... an investigator to come and investigate how the nurses treat children and mothers.'

'The nurses must have a strategy on how to address us about the shortage of medicines.

The recommendations formulated by HCWs to improve the accessibility of clinics included the need for mothers to learn to be more patient and understanding; to focus specifically on the education of pregnant women and mothers with newborn babies (at the obstetric clinic, home visits and possibly a visit to the clinic when the baby is 10 days old); to educate mothers in a group context rather than singly; and for HCWs to receive in-service training. Recommendations mentioned by only one or two HCWs, but which should not be disregarded completely, include the following: education should start at school, men should also be educated, information provided to mothers must be appropriate and mothers should be encouraged to attend clinic despite their grievances. The following quotes from the relevant primary documents illustrate the above-mentioned recommendations by HWCs:

'We tell them to be patient. There is nothing that we can do because you are working alone.'

'I think if it can (they should) before they deliver be educated on nutrition and guidelines.'

'Some of the mothers you can see are not well nourished. They are having problems. Some of them are not very clean. You wonder whether they will be able to look after their babies bygienically... All those things are supposed to be addressed by the clinic because they look at their basic guidelines. They will also be able to see if a mother is unhygienic and educate her if she have sores and let her get treatment.'

'One of the mothers came back with a 3-week-old baby. She claims that at the clinic they said they do not have time to teach her breast-feeding. She did not have money to buy formula so she is giving the baby water and cabana juice if there is any at all.'

'Currently there are no district nurses to visit mothers. They do not give the health talks to mothers. Mothers are turned back. and told not to visit the clinic after 10 days but only when the baby has to go for the first immunisation, which is after 6 weeks. I feel this is wrong.'
In-service training is needed because every year there is new information and changes on health issues especially with AIDS, TB, clinic cards, immunisation. We equip ourselves by reading. It has been a long time since we last have training. So we need proper training.'

\section{Discussion and recommendations}

The aim of this research was to contribute to the nutritional well-being of young children living in Duncan Village by investigating factors that influence clinic attendance by mothers and to formulate recommendations for optimisation of accessibility of PHC clinics in the area.

The results from the focus groups with the various categories of mothers indicate clearly that mothers in Duncan Village perceive and/or experience serious problems that make it difficult for them to attend clinic or that prevent them from doing so. These problems include both the way they are treated at the clinics (interpersonal relationships) as well as the actual services delivered. The fact that so few positive experiences were recorded indicates that the problems need to be viewed seriously. Because the mothers in the area are povertystricken, they are especially vulnerable to deciding against using health services when faced with such problems. Poverty erodes the capacity of poor people to be part of social support networks, leaving them unable to engage in and maintain social exchange ${ }^{21}$. In his concluding remarks about the results of a focus group study in Central America, Leon ${ }^{22}$ stated that the poorest sector of the population has the most difficult access to services, receives the lowest quality of care and has the fewest or no expectations. They feel themselves excluded from the system, discriminated against and treated in an undignified manner.

In our study negative experiences with service delivery elicited the most comments from participants in the focus groups. These experiences include frustration, not getting any help, having to comply with prerequisites before service is rendered (ID document and/or clinic card), disorganised services, no medicine available, being turned away, the limited service that is offered and the long waiting at the clinics. These problems with service delivery at health care institutions are not new within the South African context. Nine per cent of the 3000 adults who participated in the South African Health and Demographic Survey (SAHDS) in the Eastern Cape Province complained about the long waiting time in PHC services. The same sentiment was evident from another survey conducted in the Eastern Cape Province, where one in four people were dissatisfied with services they had received at PHC clinics 5 .

Kanani $^{20}$ emphasises that availability of adequate facilities and supplies (essential drugs, vaccines and nutrient supplements) are important determinants of the quality of care at the point of service delivery in child health programmes. The important role of availability of 
medicine at the PHC clinics in Duncan Village in ensuring client satisfaction is illustrated by the fact that a lack of medicine was the most often reported problem with services received. Leon ${ }^{22}$ also found in a study in Central America that the most basic and widespread preoccupation with health services among rural poor was how to obtain effective, cheap medication in sufficient quantities.

When the recommendations made by the mothers on what needs to be done to improve the service are considered, it is clear that almost all the comments are focused on the behaviour of health workers towards them (interpersonal relationships). This could indicate that being 'treated badly' overshadows 'not receiving any service'. Fonn et $a{ }^{23}$ found similar sentiments in two studies conducted during 1998 in three South African provinces. By far the most common aspect of maternal health services that respondents commented on in these studies was the negative way in which health service staff treated patients. The existence of this problem is further supported by the fact that $15 \%$ of the respondents who participated in the 1998 SAHDS and were dissatisfied with the service received at public health facilities cited 'rude staff' as the reason for their dissatisfaction ${ }^{5}$. Interestingly enough, in the research by Fonn et $a l .{ }^{23}$, health workers described themselves as rude, uncaring and insensitive. The role of interpersonal relations in the perception of the quality of health services was also found to be very important in Guinea ${ }^{24}$. These researchers noted that the conduct of health care professionals stood out as a central element of the judgement that users made about health services. In another study in Central America health-care service users complained about a lack of attention and disrespectful attitudes, the latter being directed especially towards those of modest socio-economic status ${ }^{22}$. The problem of 'favouritism' was also frequently voiced in the latter study, indicating that it is not a phenomenon unique to Duncan Village. Health workers admitted the existence of the problem of favouritism in the study by Fonn et al. ${ }^{23}$, when they said to the researchers that they treated clients selectively, providing better treatment to educated and well-off women and men and worse treatment to illiterate or poor women.

The negative behaviour of HCWs towards patients is not always due to a lack of training. Haddad et al. ${ }^{24}$ state that HCWs have to carry the burden of a dual legacy: that of their biomedical background, which tends to focus more on the disease (case) than on the person; and that of professionals who often abuse the authority conferred upon them in the patient-caregiver relationship. According to Vlasoff and Moreno ${ }^{25}$ research by Fonn and coworkers in South Africa indicates that class, racially defined distinctions and gender inequalities result in female health providers not necessarily being more sensitive to the needs of female clients, particularly when these clients are of a lower social class. HCWs acknowledged that a lack of gender sensitivity, in spite of being women themselves, made them judgmental ${ }^{23}$. One of the HCWs was quoted as follows: "We grow up in the same society as everyone else, we are socialised to see women in a certain way and we do, ${ }^{23}$. HCWs also indicated to Fonn et l $^{23}$ that they often take less time and care with illiterate women, as they know that these women are unlikely to complain. It follows that understanding the underlying reasons why HCWs behave inappropriately towards clients is essential when solutions for improving health services and health service utilisation are sought. According to Fahlberg et $a .^{26}$, health educators can 'empower' their clients if they recognise and respect the context and reality in which they live. Only when health workers 'recognise that people are not islands' and see contextualised people and events - i.e. holistically as a person with a health problem rather than as a case - can they find themselves providing meaningful services to their clients.

Proper and respectful behaviour should lead to feelings of trust in the health care provider. Feeling 'good' about attending PHC clinics instead of, for example, the fear, frustration and distress generated by the verbal abuse experienced by mothers in this study, which led to the decision not to attend the clinic, will increase PHC clinic accessibility. If mothers and pregnant women decide not to attend clinic or do so only late in their pregnancies, the nutrition-related information that would have been accessed at clinics will be rendered inaccessible to such women. It follows that interpersonal relationships characterised by negative attitudes and behaviour that is experienced by mothers at the PHC clinics in Duncan Village could render nutrition-related education inaccessible. Therefore, for nutrition-related messages to be accessible to mothers, HCWs will have to treat them properly and with respect, which is described by Knowles (cited in Vella ${ }^{27}$ ) as the prime factor in adult learning. If the learner does not feel respected by the teacher, 'she will not learn what she might learn'.

When considering the problems from HCWs' point of view it is clear that the main problem they experience is a heavy workload, which could keep them from giving appropriate education to clients. Staff shortages in PHC clinics in Duncan Village may be a real problem, as it was mentioned in the 2002 annual reports of all three PHC clinics in the area as a serious problem that needs to be addressed urgently ${ }^{6}$. However, if the recommendation by Lehman and Sanders ${ }^{28}$ of a workload of 25 patients per 8hour shift, which is usually recommended is 'a reasonable if not too generous' ratio, is considered, it could be argued that the workload of 24 patients per nurse per day in the clinics in Duncan Village 5 should enable the workers to provide clients with adequate services. However, it must be borne in mind that the workload that HCWs actually handle at different PHC facilities does vary due to additional tasks such as having to clean the clinic themselves, doing all clerical work or assisting HIV- 
positive patients with their social assistance grant applications that are done by some $\mathrm{HCWs}^{28}$. Another aspect commented on by Mahlalela et al. ${ }^{5}$ is that the number of personnel assigned to a facility does not always assure adequate levels of staff. A 1999 survey in the Eastern Cape found 33\% of staff absent on the day of the survey. 'Absent' staff may be attending training courses or meetings, be on maternity leave or have other legitimate reasons for not being at work ${ }^{5}$. Lehman and Sanders ${ }^{28}$ stated that the human resources sector of the health services 'holds the key' to tackling the challenges facing the health sector and it is important that their problems be addressed. The relevance of this comment to the Duncan Village clinic situation is supported by the number of recommendations made by mothers and pregnant women about the need to attend to staff shortages at the clinics. The recommendations by the HCWs themselves that they need in-service training and better co-operation with other role players, as well as the help of trained volunteers, and that they should rather educate mothers in group context and need to be more patient and understanding themselves, should also receive attention.

Problems with mothers, the clients of the PHC clinics, did not seem to feature prominently in problems mentioned by HCWs or in their recommendations on how the clinics' services could be improved. The one aspect that does seem to need attention is the problem of 'missing mothers', i.e. the fact that many mothers do not return to the clinic for further immunisations and education. It can be argued that once the problems mothers experience with the treatment and services they receive at clinics have been addressed, the problem of poor clinic attendance will disappear. However, the reality of migrating mothers and mothers who remain unmotivated to attend clinic despite possible improvements cannot be ignored.

Based on the outcomes of this research, the following recommendations were formulated to contribute to the establishment of clinics as user-friendly services, thus optimising accessibility of PHC clinics in the Duncan Village area.

1. HCWs at PHC clinics must be made aware of the important role that the clinics, and by implication they themselves, play in ensuring the nutritional well-being of young children - even if no physical help in the form of food can be provided, help in the form of advice and guidance is equally important. They should also consider that antenatal clinics are an important starting point for education and that mothers must also be encouraged to visit clinics as early as possible after the birth of the baby; that special attention must be given to young mothers who seem vulnerable; and that the long waiting periods at clinics can be used to educate mothers.

2. HCWs need to be empowered to render an effective service by the following: providing in-service training to ensure optimal competence; training in the principles of good communication and human interaction to improve the $\mathrm{HCW}$-client interaction; and training to ensure the use of sound counselling practices and adult education principles when educating mothers.

3. HCWs should always remember that their clients are part of a larger reality that includes physical, biological, psychological, spiritual, historical, cultural, social, economic and political dimensions; they should also understand how gender issues affect their clients' lives, as this can lead to positive changes in the relationship between HCWs and their clients.

4. The local government should give attention to the workload problems experienced by the HCWs and ensure the provision of sufficient funds and supplies, especially vaccines and medicines, to run the services effectively.

5. The possibility of involving the community (volunteers) in establishing the clinics as user-friendly services should be investigated; community-based drives to motivate mothers to attend the clinic should also be investigated; those who are ultimately not reached by the PHC clinics, despite the improvement of accessibility, should not be forgotten. Efforts to reach them should never be abandoned.

\section{References}

1 World Health Organization (WHO). Strategic Directions for Improving the Health and Development of Children and Adolescents. Geneva: WHO, 2002.

2 United Nations Administrative Committee on Coordination, Sub-committee on Nutrition (ACC/SCN). Fourth Report on the World Nutrition Situation. Geneva: ACC/SCN in collaboration with International Food Policy Research Institute, 2000.

3 South African Vitamin A Consultative Group (SAVACG). Children Aged 6 to 71 months in South Africa, 1994: Their Anthropometric, Vitamin A and Iron Status. Medical Update, Vitamin Information Centre, No. 26, Parts I and II. Johannesburg: SAVACG, 1996.

4 Labadarios D, ed. The National Food Consumption Survey (NFCS): Children aged 1-9 years. Stellenbosch: Department of Health, 2000.

5 Mahlalela X, Rohde J, Meidany F, Hutchinson P, Bennett J. Primary Health Care in the Eastern Cape Province: 19972000. East London: Department of Health, 2001

6 Buffalo City Municipality. Buffalo City Municipality Primary Health Care Report. East London: Buffalo City Municipality, 2003.

7 Bank L, Jekwa M, Lujabe P, Mlomo B. Small Business Development in Duncan Village: Towards a New Development Framework. East London: Institute of Social and Economic Research, Rhodes University, 1996.

8 Chelala C. Environmental Impact on Child Health. Washington, DC: Pan American Health Organization, 2000.

9 United Nations Children's Fund (UNICEF). The State of the World's Children 1998. New York: Oxford University Press for UNICEF, 1997.

10 De Villiers A, Senekal M. Determinants of growth failure in 12-24-month-old children in a high-density urban slum 
community in East London, South Africa. European Journal of Clinical Nutrition 2002; 56: 1231-41.

11 United Nations Children's Fund (UNICEF). UNICEF's Priorities for Children 2002-2005. New York: UNICEF, 2002

12 United Nations Children's Fund (UNICEF). Baby Friendly and Beyond: Integrated Care for Mother and Child. A publication of the Baby-Friendly Hospital Initiative. New York: UNICEF, April/June 2002.

13 Allen LH, Gillespie SR. What Works? A Review of the Efficacy and Effectiveness of Nutrition Interventions. Geneva: United Nations Administrative Committee on Coordination, Subcommittee on Nutrition in collaboration with the Asian Development Bank, 2001.

14 Shearley AE. The societal value of vaccination in developing countries. Vaccine 1999; 17: S109-12.

15 United Nations Children's Fund (UNICEF). Strategy for Improved Nutrition of Children and Women in Developing Countries. Policy Review Paper E/CEF/1990/1.6. New York: UNICEF, 1990; 5-36.

16 Dickin K, Griffiths M, Piwoz E. Designing by Dialogue: A Program Planners' Guide to Consultative Research for Improving Young Child Feeding. Support for Analysis and Research in Africa. Washington, DC: US Agency for International Development, Bureau for Africa, Office of Sustainable Development, 1997.

17 Fern EF. Advanced Focus Group Research. Thousand Oaks, CA: Sage, 2001.

18 Babbie E, Mouton J. The Practice of Social Research. Cape Town: Oxford University Press, 2001.

19 Muhr T. Atlas/ti: Short User's Manual. Berlin: Scientific Software Development, 1997.
20 Kanani SJ. Introducing participatory research to university and government health systems: some experiences from India. In: de Koning K, Martin M, eds. Participatory Research in Health: Issues and Experiences. Johannesburg: National Progressive Primary Health Care Network, 1996.

21 De La Rocha GM, Grinspun A. Private adjustments: households, crisis and work. In: Grinspun A, ed. Choices for the Poor: Lessons from National Poverty Strategies. New York: United Nations Development Programme, 2001.

22 Leon M. Perceptions of health care quality in Central America. International Journal for Quality in Health Care 2003; 15: 66-71.

23 Fonn S, Xaba M, Tint K, Varkey S. Maternal health services in South Africa. South African Medical Journal 1998; 88 697-702.

24 Haddad S, Fournier P, Machouf N, Yatara F. What does quality mean to lay people? Community perceptions of primary health care services in Guinea. Social Science \& Medicine 1998; 47: 381-94.

25 Vlassoff C, Moreno CG. Placing gender at the centre of health programming: challenges and limitations. Social Science \& Medicine 2002; 54: 1713-23.

26 Fahlberg LL, Poulin AM, Girdano DA, Dusek DE. Empowerment as an emerging approach in health education. Journal of Health Education 1991; 22: 185-93.

27 Vella J. Learning to listen/learning to teach: training trainers in the principles and practices of popular education. Convergence 1994; 27: 5-21.

28 Lehman U, Sanders D. Human resources development. In: South African Health Review. Durban: Health Systems Trust, 2002. 